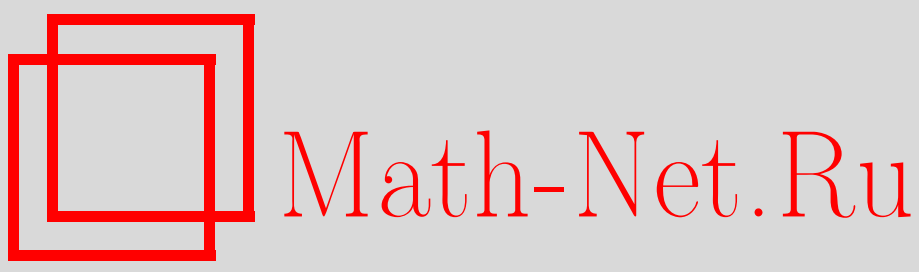

М. Е. Чанга, О числах, все простые делители которых лежат в специальных промежутках, Изв. РАН. Сер. матем., 2003, том 67, выпуск 4, 213-224

DOI: https://doi.org/10.4213/im447

Использование Общероссийского математического портала Math-Net.Ru подразумевает, что вы прочитали и согласны с пользовательским соглашением http://www. mathnet.ru/rus/agreement

Параметры загрузки:

IP: 18.208 .226 .222

26 апреля 2023 г., $16: 28: 39$ 
УДК 511

\author{
М. Е. Чанга
}

\title{
О числах, все простые делители которых лежат в специальных промежутках
}

\begin{abstract}
Изучается распределение чисел, все простые делители которых лежат в специальных промежутках, и по таким числам суммируются различные мультипликативные функции. Для этих сумматорных функций получены асимптотические формулы с главным членом, имеющим вид суммы с растущим числом слагаемых. Показано, что эта сумма с точностью до первого отброшенного члена приближается конечным числом своих слагаемых. Кроме того, обсуждается эффект обращения главного члена подобных асимптотических формул в конечную сумму при определенных соотношениях параметров задачи.
\end{abstract}

Библиограффия: 10 наименований.

Пусть заданы нецелое положительное число $a$ и натуральное число $D$, не меньшее двух. При любом натуральном $l$ с условиями $1 \leqslant l \leqslant D$ определим множество

$$
E_{l}=\bigcup_{n=0}^{\infty}\left[(D n+l-1)^{1 / a},(D n+l)^{1 / a}\right) .
$$

Если через $\delta_{l}(x)$ обозначить характеристическую функцию множества $E_{l}$ и положить

$$
\sum_{p \leqslant x} \delta_{l}(p)=\frac{\pi(x)}{D}+r_{l}(x),
$$

то для $r_{l}(x)$ имеет место оценка

$$
r_{l}(x) \ll x^{1-\Delta}
$$

с некоторым положительным $\Delta$, зависяшим от $a$. Для случая $0<a<1$ такая оценка была получена И. М. Виноградовым [1]; случай нецелых $a$, бо́льших единицы, разобран в работах [2]-[4].

Для каждого $l$ определим в полуплоскости $\operatorname{Re} s>1$ функцию

$$
F_{l}(s)=\prod_{p \in E_{l}}\left(1-\frac{1}{p^{s}}\right)^{-1} .
$$

В настоящей статье используем принятое обозначение $s=\sigma+i t$. Пусть $M_{l}-$ множество натуральных чисел, в каноническое разложение которых входят только простые из $E_{l}$. Тогда при $\operatorname{Re} s>1$ имеем

$$
F_{l}(s)=\sum_{n \in M_{l}} \frac{1}{n^{s}} .
$$


Это равенство доказывается аналогично известному тождеству Эйлера для дзета-функции Римана.

Настоящая работа посвящена изучению некоторых свойств чисел множества $M_{l}$, в частности суммированию значений мультипликативных функций по числам из таких множеств. Нам понадобятся два вспомогательных утверждения. (В дальнейшем $c, c_{1}, c_{2}, \ldots$ - положительные постоянные.)

ЛЕмма 1. В области $\operatorname{Re} s>1-\Delta$, разрезанной вдоль горизонтальных отрезков, имеющих правым концом точку $s=1$ или нетривиальный нуль дзета-функиии Римана, справедливо равенство

$$
F_{l}(s)=\sqrt[D]{\zeta(s)} e^{G_{l}(s)},
$$

где $\sqrt[D]{\zeta(s)}$ положителен при $s>1$, а функиия

$$
G_{l}(s)=s \int_{2}^{\infty} \frac{r_{l}(u) d u}{u\left(u^{s}-1\right)}
$$

аналитична в области $\operatorname{Re} s>1-\Delta$. Кроме того, при $T \geqslant 10$ в области $\sigma \geqslant 1-c_{1} / \ln T,|t| \leqslant T$ нет нулей дзета-функции Римана, а на границе этой области имеет место оценка

$$
(\ln T)^{-c_{2}} \ll\left|F_{l}(s)\right| \ll(\ln T)^{c_{2}} .
$$

ДоКАЗАТЕЛЬСТВО. В силу оценки (1) для $r_{l}(x)$ несобственный интеграл, представляющий функцию $G_{l}(s)$, сходится равномерно внутри области $\operatorname{Re} s>1-\Delta$. Следовательно, функция $G_{l}(s)$ аналитична в полуплоскости $\operatorname{Re} s>1-\Delta$.

Пользуясь преобразованием Абеля, при любом натуральном $k$ находим

$$
\sum_{p \leqslant x} \frac{\delta_{l}(p)}{p^{k s}}=\frac{1}{x^{k s}} \sum_{p \leqslant x} \delta_{l}(p)+k s \int_{2}^{x} \sum_{p \leqslant u} \delta_{l}(p) \frac{d u}{u^{k s+1}} .
$$

С другой стороны, имеем

$$
\frac{1}{D} \sum_{p \leqslant x} \frac{1}{p^{k s}}=\frac{1}{x^{k s}} \frac{\pi(x)}{D}+k s \int_{2}^{x} \frac{\pi(u)}{D} \frac{d u}{u^{k s+1}} .
$$

Вычитая второе равенство из первого, получим

$$
\sum_{p \leqslant x} \frac{\delta_{l}(p)}{p^{k s}}-\frac{1}{D} \sum_{p \leqslant x} \frac{1}{p^{k s}}=\frac{r_{l}(x)}{x^{k s}}+k s \int_{2}^{x} r_{l}(u) \frac{d u}{u^{k s+1}} .
$$

Разделим это равенство на $k$ и просуммируем по всем натуральньм $k$. В полуплоскости $\operatorname{Re} s>0$ это даст

$$
\begin{array}{r}
\sum_{p \leqslant x} \delta_{l}(p) \ln \left(1-\frac{1}{p^{s}}\right)^{-1}-\frac{1}{D} \sum_{p \leqslant x} \ln \left(1-\frac{1}{p^{s}}\right)^{-1} \\
=r_{l}(x) \ln \left(1-\frac{1}{x^{s}}\right)^{-1}+s \int_{2}^{x} \frac{r_{l}(u) d u}{u\left(u^{s}-1\right)}
\end{array}
$$


где $\ln \left(1-1 / x^{s}\right)^{-1}$ положителен при $s>0$. Здесь мы пользуемся тем, что

$$
\sum_{k=1}^{\infty} \int_{2}^{x} r_{l}(u) \frac{d u}{u^{k s+1}}=\int_{2}^{x} \frac{r_{l}(u)}{u} \sum_{k=1}^{\infty} u^{-k s} d u=\int_{2}^{x} \frac{r_{l}(u) d u}{u\left(u^{s}-1\right)} .
$$

Перемена порядка суммирования и интегрирования обосновывается равномерной сходимостью ряда на отрезке $[2, x]$.

В полуплоскости $\operatorname{Re} s>1$, потенцируя полученное равенство и переходя к пределу при $x \rightarrow \infty$, получим искомое представление $F_{l}(s)$. Согласно принципу аналитического продолжения это представление распространяется на указанную в условии область.

Рассмотрим при $T \geqslant 10$ область $\sigma \geqslant 1-\Delta / \ln T,|t| \leqslant T$. Выберем параметр $A=T^{1 / \Delta}$ и разобьем интеграл, задающий $G_{l}(s)$, на два. Пользуясь оценкой $(1)$ для $r_{l}(x)$, оценим интеграл по промежутку $[A, \infty)$ тривиально:

$$
s \int_{A}^{\infty} \frac{r_{l}(u) d u}{u\left(u^{s}-1\right)} \ll T \int_{A}^{\infty} u^{-1-\Delta(1-1 / \ln T)} d u \ll 1 .
$$

Для оценки интеграла по промежутку $[2, A]$ используем соотношение $(2)$ при $x=A$ :

$$
s \int_{2}^{A} \frac{r_{l}(u) d u}{u\left(u^{s}-1\right)} \ll A^{-\Delta(1-1 / \ln T)}+\sum_{p \leqslant A} p^{-1+\Delta / \ln T} \ll \ln \ln T .
$$

Таким образом, в рассматриваемой области имеем

$$
G_{l}(s) \ll \ln \ln T \text {. }
$$

Согласно теореме Валле-Пуссена о гранище нулей $\zeta(s)$, а также теоремам об оценке $\zeta(s)$ и $1 / \zeta(s)$ в окрестности единичной прямой $[5$, c. 54,65$]$ найдется положительное число $c_{1}<\Delta$ такое, что в области $\sigma \geqslant 1-c_{1} / \ln T,|t| \leqslant T$ не будет нулей $\zeta(s)$, а на ее гранище будут вьполняться оценки

$$
\zeta(s) \ll \ln T, \quad \frac{1}{\zeta(s)} \ll \ln T .
$$

Справедливость первой оценки при малых $t$ вытекает из наличия у функции $\zeta(s)$ полюса первого порядка в точке $s=1$. Комбинируя эти оценки с оценкой (3) для $G_{l}(s)$, получим требуемые неравенства для $F_{l}(s)$. Это и завершает доказательство.

Лемма 2. Пусть функиия $H(s)$ аналитична в круге $|s|<\Delta$ и $A_{k}$ обозначает ее $k$-й коэффициент Тейлора в этом круге. Пусть контур Г начинается в точке $-(\ln x)^{-1 / 2}-i 0$, обходит начало координат против часовой стрелки и заканчивается в точке $-(\ln x)^{-1 / 2}+i 0$. Пусть

$$
I(x)=\frac{1}{2 \pi i} \int_{\Gamma} x^{s} s^{-\frac{\alpha}{D}} H(s) d s
$$


где $\alpha$ - фиксированное челое число и $s^{-\alpha / D}$ положительно при $s>0$. Тогда имеет место асимптотическая формула

$$
I(x)=\sum_{0 \leqslant n \leqslant \sqrt{\ln x}} A_{n} \frac{(\ln x)^{\frac{\alpha}{D}-n-1}}{\Gamma\left(\frac{\alpha}{D}-n\right)}+O\left(e^{-c_{3} \sqrt{\ln x}}\right) .
$$

Кроме того, при любом фиксированном челом $N$

$$
\sum_{N \leqslant n \leqslant \sqrt{\ln x}}\left|\frac{A_{n}}{\Gamma\left(\frac{\alpha}{D}-n\right)}\right|(\ln x)^{\frac{\alpha}{D}-n-1} \ll(\ln x)^{\frac{\alpha}{D}-N-1} .
$$

ДоКАЗАТЕЛЬСТВо. Пусть $\delta$ - фиксированное положительное число, меньшее $\Delta$. Обозначим через $M$ максимум модуля $H(s)$ на окружности $|s|=\delta$. Согласно неравенствам Коши при любом $n$ имеет место оценка

$$
\left|A_{n}\right| \leqslant M \delta^{-n}
$$

При больших $x$ можно полагать, что контур Г целиком лежит в круге $|s| \leqslant \delta$. В силу равномерной сходимости ряда Тейлора $H(s)$ в этом круге получаем

$$
I(x)=\sum_{n=0}^{\infty} A_{n} \frac{1}{2 \pi i} \int_{\Gamma} x^{s} s^{n-\frac{\alpha}{D}} d s=\sum_{n=0}^{\infty} A_{n} I_{n}(x) .
$$

Пусть $n>\alpha / D+\sqrt{\ln x}$. В этом случае

$$
\left|I_{n}(x)\right| \leqslant \frac{1}{\pi} \int_{0}^{(\ln x)^{-1 / 2}} x^{-u} u^{n-\frac{\alpha}{D}} d u
$$

На отрезке интегрирования подынтегральная функция монотонно возрастает, поэтому

$$
\left|I_{n}(x)\right| \leqslant \frac{1}{\pi} e^{-\sqrt{\ln x}}(\ln x)^{\frac{1}{2}\left(\frac{\alpha}{D}-n-1\right)} .
$$

Отсюда, пользуясь оценкой (4), при некотором $c_{3}<1$ находим

$$
\sum_{n>\alpha / D+\sqrt{\ln x}} A_{n} I_{n}(x) \ll e^{-c_{3} \sqrt{\ln x}} \sum_{n>\alpha / D+\sqrt{\ln x}}(\delta \sqrt{\ln x})^{-n}
$$

Пусть теперь $n \leqslant \alpha / D+\sqrt{\ln x}$. Дополним контур $\Gamma$ до контура $\Gamma^{\prime}$ двумя лучами, идушими от конщов Г вдоль отрицательной части вещественной оси. Из интегрального представления Ханкеля для гамма-функции [6, с. 28] имеем

$$
\frac{1}{2 \pi i} \int_{\Gamma^{\prime}} x^{s} s^{n-\frac{\alpha}{D}} d s=\frac{(\ln x)^{\frac{\alpha}{D}-n-1}}{\Gamma\left(\frac{\alpha}{D}-n\right)} .
$$


Таким образом, можно записать

$$
I_{n}(x)=\frac{(\ln x)^{\frac{\alpha}{D}-n-1}}{\Gamma\left(\frac{\alpha}{D}-n\right)}+R_{n}(x)
$$

где

$$
\left|R_{n}(x)\right| \leqslant \frac{1}{\pi} \int_{(\ln x)^{-1 / 2}}^{\infty} x^{-u} u^{n-\frac{\alpha}{D}} d u .
$$

Теперь необходимо оценить последний интеграл. Если $n \leqslant \alpha / D$, то функция $u^{n-\alpha / D}$ монотонно убывает. Поэтому

$$
\left|R_{n}(x)\right| \leqslant \frac{1}{\pi}(\ln x)^{\frac{1}{2}\left(\frac{\alpha}{D}-n\right)} \int_{(\ln x)^{-1 / 2}}^{\infty} x^{-u} d u=\frac{1}{\pi} e^{-\sqrt{\ln x}}(\ln x)^{\frac{1}{2}\left(\frac{\alpha}{D}-n-2\right)} .
$$

Если $n>\alpha / D$, то повторным интегрированием по частям можно свести этот случай к предыдушему. Имеем

$$
\begin{aligned}
\left|R_{n}(x)\right| \leqslant & \frac{1}{\pi} e^{-\sqrt{\ln x}}(\ln x)^{\frac{1}{2}\left(\frac{\alpha}{D}-n-2\right)} \\
& \times\left[1+\frac{n-\frac{\alpha}{D}}{\sqrt{\ln x}}+\cdots+\frac{\left(n-\frac{\alpha}{D}\right) \cdots\left(n-\frac{\alpha}{D}-\left[n-\frac{\alpha}{D}\right]\right)}{(\sqrt{\ln x})^{[n-\alpha / D]+1}}\right] \\
\leqslant & \frac{1}{\pi} e^{-\sqrt{\ln x}}(\ln x)^{\frac{1}{2}\left(\frac{\alpha}{D}-n-2\right)} \\
& \times\left[1+\frac{n-\frac{\alpha}{D}}{\sqrt{\ln x}}+\left(\frac{n-\frac{\alpha}{D}}{\sqrt{\ln x}}\right)^{2}+\cdots+\left(\frac{n-\frac{\alpha}{D}}{\sqrt{\ln x}}\right)^{\left[n-\frac{\alpha}{D}\right]+1}\right] .
\end{aligned}
$$

Учитывая, что $n \leqslant \alpha / D+\sqrt{\ln x}$, находим

$$
R_{n}(x) \ll e^{-\sqrt{\ln x}}(\ln x)^{\frac{1}{2}\left(\frac{\alpha}{D}-n-1\right)},
$$

откуда, вновь прибегая к оценке (4), получаем

$$
\sum_{0 \leqslant n \leqslant \alpha / D+\sqrt{\ln x}} A_{n} R_{n}(x) \ll e^{-c_{3} \sqrt{\ln x}} \sum_{0 \leqslant n \leqslant \alpha / D+\sqrt{\ln x}}(\delta \sqrt{\ln x})^{-n} .
$$

Объединяя эту оценку с соотношением (5), при достаточно больших $x$ находим

$$
I(x)=\sum_{0 \leqslant n \leqslant \alpha / D+\sqrt{\ln x}} A_{n} \frac{(\ln x)^{\frac{\alpha}{D}-n-1}}{\Gamma\left(\frac{\alpha}{D}-n\right)}+O\left(e^{-c_{3} \sqrt{\ln x}}\right) .
$$

Используя формулы дополнения и понижения для гамма-функции, при $n>$ $\alpha / D+1$ имеем

$$
\frac{1}{\left|\Gamma\left(\frac{\alpha}{D}-n\right)\right|} \leqslant \frac{1}{\pi}\left|\Gamma\left(n+1-\frac{\alpha}{D}\right)\right| \leqslant \frac{1}{\pi}\left(n-\frac{\alpha}{D}\right)^{\left[n-\frac{\alpha}{D}\right]} \leqslant \frac{1}{\pi}\left(n-\frac{\alpha}{D}\right)^{n-\frac{\alpha}{D}}
$$


Таким образом, при $n \asymp \sqrt{\ln x}$ справедлива оценка

$$
A_{n} \frac{(\ln x)^{\frac{\alpha}{D}-n-1}}{\Gamma\left(\frac{\alpha}{D}-n\right)} \ll e^{-c_{4} \sqrt{\ln x} \ln \ln x}
$$

так что добавление или отбрасывание в сумме (6) конечного числа слагаемых c $n \asymp \sqrt{\ln x}$ не повлияет на остаток. Далее, общий член суммы (6) при $n>\alpha / D+1$ оценивается следуюшим образом:

$$
\left|A_{n} \frac{(\ln x)^{\frac{\alpha}{D}-n-1}}{\Gamma\left(\frac{\alpha}{D}-n\right)}\right| \leqslant \frac{M \delta^{-\frac{\alpha}{D}}}{\pi \ln x}\left(\frac{n-\frac{\alpha}{D}}{\delta \ln x}\right)^{n-\frac{\alpha}{D}}
$$

причем функция в правой части монотонно убывает при $n$, заключенном в пределах от $\alpha / D+1$ до $\sqrt{\ln x}$, если $x$ достаточно велико. Пусть $N$ - фиксированное целое неотрицательное число. Не ограничивая общности, можно считать, что $N>\alpha / D$. В этом случае справедлива оценка

$$
\begin{aligned}
& \sum_{N+1 \leqslant n \leqslant \sqrt{\ln x}}(\ln x)^{\frac{\alpha}{D}-n-1}\left|\frac{A_{n}}{\Gamma\left(\frac{\alpha}{D}-n\right)}\right| \\
& \leqslant \frac{M \delta^{-\frac{\alpha}{D}}}{\pi \sqrt{\ln x}}\left(\frac{N+1-\frac{\alpha}{D}}{\delta \ln x}\right)^{N+1-\frac{\alpha}{D}} \ll(\ln x)^{\frac{\alpha}{D}-N-\frac{3}{2}} .
\end{aligned}
$$

Добавляя сюда оценку $N$-го слагаемого, получаем второе утверждение леммы. Этим доказательство завершается.

ЗАМЕЧАнИЕ 1 . Если $\alpha / D$ - целое число, то главный член асимптотики обращается в конечную сумму по $n \leqslant \alpha / D-1$. Это будет точное равенство, так как в данном случае подынтегральная функция однозначна и интеграл $I(x)$ равен ее вычету в точке $s=0$.

Для любого натурального $n$ однозначно определены $D$ натуральных чисел $n_{1}, \ldots, n_{D}$ таких, что $n=n_{1} \ldots n_{D}, n_{i} \in M_{i}$. Пусть $\tau_{k}(n)$ обозначает число представлений натурального $n$ произведением $k$ натуральных чисел, причем $\tau_{0}(n)=0$ при $n>1$ и $\tau_{0}(1)=1$, так как "пустое" произведение считается равным единице. Пусть заданы $D$ целых неотрицательных чисел $\alpha_{1}, \ldots, \alpha_{D}$, не все равные нулю, и $\alpha=\alpha_{1}+\cdots+\alpha_{D}$. Рассмотрим функцию

$$
\omega(n)=\tau_{\alpha_{1}}\left(n_{1}\right) \ldots \tau_{\alpha_{D}}\left(n_{D}\right)
$$

Обозначим через $\Omega(x)$ сумматорную функцию $\omega(n)$. Основным результатом настоящей работы является вывод асимптотической формулы для $\Omega(x)$.

TEOPEMA 1. Пусmb

$$
H(s)=\frac{1}{s}((s-1) \zeta(s))^{\frac{\alpha}{D}} e^{\alpha_{1} G_{1}(s)+\cdots+\alpha_{D} G_{D}(s)}
$$


причем $H(s)$ положительна при $s>1, A_{n}$ обозначает $n$-й коэффициент ее разложения Тейлора с чентром в точке $s=1$. Тогда имеет место асимптотическая формула

$$
\Omega(x)=\sum_{n \leqslant x} \omega(n)=x \sum_{0 \leqslant n \leqslant \sqrt{\ln x}} A_{n} \frac{(\ln x)^{\frac{\alpha}{D}-n-1}}{\Gamma\left(\frac{\alpha}{D}-n\right)}+O\left(x e^{-c \sqrt{\ln x}}\right) .
$$

Кроме того, при любом фиксированном целом $N$

$$
\Omega(x)=x \sum_{0 \leqslant n \leqslant N} A_{n} \frac{(\ln x)^{\frac{\alpha}{D}-n-1}}{\Gamma\left(\frac{\alpha}{D}-n\right)}+O\left(x(\ln x)^{\frac{\alpha}{D}-N-2}\right) .
$$

ДоКАЗАТЕЛЬСТво. Функция $\omega(n)$ есть число представлений натурального $n$ произведением $\alpha$ чисел, первые $\alpha_{1}$ из которых принадлежат множеству $M_{1}$, следуюшие $\alpha_{2}$ чисел - множеству $M_{2}$ и т.д. Соответственно, производящая функция для $\omega(n)$ при $\operatorname{Re} s>1$ имеет вид

$$
F(s)=\sum_{n=1}^{\infty} \frac{\omega(n)}{n^{s}}=F_{1}^{\alpha_{1}}(s) \ldots F_{D}^{\alpha_{D}}(s)
$$

Используя неравенство Марджанишвили [7, с. 60], находим

$$
\Omega(x)=\sum_{n \leqslant x} \tau_{\alpha_{1}}\left(n_{1}\right) \ldots \tau_{\alpha_{D}}\left(n_{D}\right) \leqslant \sum_{n \leqslant x} \tau_{\alpha}(n) \ll x \ln ^{\alpha-1} x .
$$

Отсюда, интегрируя по частям, при $b=1+1 / \ln x$ получаем

$$
\int_{1}^{\infty} \frac{\Omega(x)}{x^{b+1}} d x \ll \int_{1}^{\infty} \frac{\ln ^{\alpha-1} x}{x^{b}} d x \ll(b-1)^{-\alpha}=\ln ^{\alpha} x .
$$

Используя общую теорему о связи между суммой коэффициентов ряда Дирихле и функцией, задаваемой этим рядом $[7$, с. 82$]$, имеем

$$
\int_{1}^{x} \Omega(u) d u=\frac{1}{2 \pi i} \int_{b-i T}^{b+i T} \frac{F(s) x^{s+1}}{s(s+1)} d s+O\left(x^{2} e^{-c_{5} \sqrt{\ln x}}\right),
$$

где $T=e^{c_{1} \sqrt{\ln x}}, c_{1}-$ постоянная из леммы 1.

Введем параметр $\beta=1-c_{1} / \ln T=1-(\ln x)^{-1 / 2}$. Согласно лемме 1 функция $F(s)$ аналитически продолжается левее единичной прямой, причем в области $\sigma \geqslant \beta,|t| \leqslant T$ она не имеет особенностей, за исключением точки ветвления при $s=1$. Согласно теореме Коши можно деформировать вертикальный отрезок интегрирования в контур $C$, состояший из отрезков, последовательно соединяющих точки $b-i T, \beta-i T$ и $\beta-i 0$, петли $\Gamma$, обходящей точку $s=1$ против часовой 
стрелки, и отрезков, последовательно соединяющих точки $\beta+i 0, \beta+i T$ и $b+i T$. Таким образом получим

$$
\frac{1}{2 \pi i} \int_{b-i T}^{b+i T} \frac{F(s) x^{s+1}}{s(s+1)} d s=\frac{1}{2 \pi i} \int_{\Gamma} \frac{F(s) x^{s+1}}{s(s+1)} d s+R_{1}(x)+R_{2}(x),
$$

где через $R_{1}(x)$ и $R_{2}(x)$ обозначены интегралы по горизонтальным и вертикальным отрезкам контура $C$ соответственно. Согласно лемме 1 на этих отрезках $F(s) \ll \ln ^{c_{6}} T$, поэтому

$$
\begin{gathered}
R_{1}(x) \ll \frac{x^{2}}{T^{2}} \ln ^{c_{6}} T \ll x^{2} e^{-c_{7} \sqrt{\ln x}}, \\
R_{2}(x) \ll x^{1+\beta} \ln ^{c_{6}} T \ll x^{2} e^{-c_{8} \sqrt{\ln x}} .
\end{gathered}
$$

Собирая вместе полученные оценки и пользуясь явным представлением $F(s)$ из леммы 1 , получаем

$$
\int_{1}^{x} \Omega(u) d u=\frac{1}{2 \pi i} \int_{\Gamma}(s-1)^{-\frac{\alpha}{D}} \frac{x^{s+1}}{s+1} H(s) d s+O\left(x^{2} e^{-c_{9} \sqrt{\ln x}}\right)
$$

где $(s-1)^{-\alpha / D}$ положительно при $s>1$, а $H(s)$ - функция из условия теоремы.

Для вычисления интеграла по Г сделаем сдвиг начала координат в точку $s=1$ и воспользуемся леммой 2 . Получим

$$
\int_{1}^{x} \Omega(u) d u=x^{2} \sum_{0 \leqslant n \leqslant \sqrt{\ln x}} B_{n} \frac{(\ln x)^{\frac{\alpha}{D}-n-1}}{\Gamma\left(\frac{\alpha}{D}-n\right)}+O\left(x^{2} e^{-c_{10} \sqrt{\ln x}}\right),
$$

где $B_{n}$ обозначает $n$-й коэффициент Тейлора $H(s) /(s+1)$ в точке $s=1$. Применяя формулу Лейбница, находим

$$
A_{n}=B_{n-1}+2 B_{n}
$$

при любом $n$, если положить $B_{-1}=0$.

Функция $\Omega(x)$ монотонно возрастает, поэтому ее асимптотику можно получить, применяя метод асимптотического дифференщирования. При $0<h<x / 2$ имеем

$$
\frac{1}{h} \int_{x-h}^{x} \Omega(u) d u \leqslant \Omega(x) \leqslant \frac{1}{h} \int_{x}^{x+h} \Omega(u) d u .
$$

Рассмотрим следуюшее выражение:

$$
\begin{aligned}
\pm \frac{1}{h}(x \pm h)^{2} & \sum_{0 \leqslant n \leqslant \sqrt{\ln (x \pm h)}} B_{n} \frac{(\ln (x \pm h))^{\frac{\alpha}{D}-n-1}}{\Gamma\left(\frac{\alpha}{D}-n\right)} \\
& \mp \frac{1}{h} x^{2} \sum_{0 \leqslant n \leqslant \sqrt{\ln x}} B_{n} \frac{(\ln x)^{\frac{\alpha}{D}-n-1}}{\Gamma\left(\frac{\alpha}{D}-n\right)} \\
= & \pm \frac{1}{h} \int_{1}^{x \pm h} \Omega(u) d u \mp \frac{1}{h} \int_{1}^{x} \Omega(u) d u+O\left(x^{2} h^{-1} e^{-c_{10} \sqrt{\ln x}}\right) .
\end{aligned}
$$


Согласно оценке (7) можно заменить верхний предел изменения $n$ в первой сумме на $\sqrt{\ln x}$. В дальнейшем, используя подобные соображения, мы не будем оговаривать это особо. Применим к первой сумме формулу Тейлора с остаточным членом в форме Лагранжа. Получим

$$
\begin{aligned}
& \pm \frac{1}{h} \int_{1}^{x \pm h} \Omega(u) d u \mp \frac{1}{h} \int_{1}^{x} \Omega(u) d u+O\left(x^{2} h^{-1} e^{-c_{10} \sqrt{\ln x}}\right) \\
& \quad=x \sum_{0 \leqslant n \leqslant \sqrt{\ln x}} B_{n}\left(\frac{2(\ln x)^{\frac{\alpha}{D}-n-1}}{\Gamma\left(\frac{\alpha}{D}-n\right)}+\frac{\left(\frac{\alpha}{D}-n-1\right)(\ln x)^{\frac{\alpha}{D}-n-2}}{\Gamma\left(\frac{\alpha}{D}-n\right)}\right)+R
\end{aligned}
$$

где

$$
R \ll h \sum_{0 \leqslant n \leqslant \sqrt{\ln x}}\left|\frac{B_{n}}{\Gamma\left(\frac{\alpha}{D}-n\right)}\right|(\ln (x+\theta h))^{\frac{\alpha}{D}-n-1} \ll h(\ln x)^{\frac{\alpha}{D}-1} .
$$

При выделении главной части второй производной мы пользовались тем, что $n \leqslant$ $\sqrt{\ln x}$. Последняя оценка вытекает из второго утверждения леммы 2. Теперь воспользуемся формулой понижения для гамма-функции и соотношением (8), связывающим коэффициенты $A_{n}$ и $B_{n}$. Получим следующее равенство:

$$
\begin{aligned}
& \pm \frac{1}{h} \int_{1}^{x \pm h} \Omega(u) d u \mp \frac{1}{h} \int_{1}^{x} \Omega(u) d u \\
& \quad=x \sum_{0 \leqslant n \leqslant \sqrt{\ln x}} A_{n} \frac{(\ln x)^{\frac{\alpha}{D}-n-1}}{\Gamma\left(\frac{\alpha}{D}-n\right)}+O\left(x^{2} h^{-1} e^{-c_{10} \sqrt{\ln x}}+h(\ln x)^{\frac{\alpha}{D}-1}\right) .
\end{aligned}
$$

Выбирая параметр $h=x e^{-c_{10} \sqrt{\ln x} / 2}$, мы видим, что верхняя и нижняя оценки для $\Omega(x)$ асимптотически совпадают. Отсюда получим искомую асимптотическую формулу для $\Omega(x)$. Доказательство завершается применением второго утверждения леммы 2.

ЗАмЕчАниЕ 2. Подобным образом можно получить аналогичные асимптотические формулы в случае других функций, отличающихся от $\omega(n)$ заменой одного или нескольких $\tau$-множителей на мультипликативнье функции, производяшие функции которых выражаются произведением дзета-функций.

Получим некоторые следствия доказанной теоремы. Пусть заданы $k$ различных натуральных чисел $l_{1}, \ldots, l_{k}$ таких, что выполнены условия $1 \leqslant l_{i} \leqslant D$. Пусть $E=E_{l_{1}} \cup \cdots \cup E_{l_{k}}$ и $M$ - множество натуральных чисел, все простые делители которых принадлежат множеству $E$.

СлЕДСТВИЕ 1. Имеет место асимптотическая формула

$$
\sum_{\substack{n \leqslant x \\ n \in M}} 1=x \sum_{0 \leqslant n \leqslant \sqrt{\ln x}} A_{n} \frac{(\ln x)^{\frac{k}{D}-n-1}}{\Gamma\left(\frac{k}{D}-n\right)}+O\left(x e^{-c \sqrt{\ln x}}\right) .
$$

Кроме того, при любом фиксированном челом $N$

$$
\sum_{\substack{n \leqslant x \\ n \in M}} 1=x \sum_{0 \leqslant n \leqslant N} A_{n} \frac{(\ln x)^{\frac{k}{D}-n-1}}{\Gamma\left(\frac{k}{D}-n\right)}+O\left(x(\ln x)^{\frac{k}{D}-N-2}\right) .
$$


СлЕДСТВИЕ 2. Имеет место асимптотическая формула

$$
\sum_{\substack{n \leqslant x \\ n \in M}} \tau(n)=x \sum_{0 \leqslant n \leqslant \sqrt{\ln x}} B_{n} \frac{(\ln x)^{\frac{2 k}{D}-n-1}}{\Gamma\left(\frac{2 k}{D}-n\right)}+O\left(x e^{-c \sqrt{\ln x}}\right) .
$$

Кроме того, при любом фиксированном иелом $N$

$$
\sum_{\substack{n \leqslant x \\ n \in M}} \tau(n)=x \sum_{0 \leqslant n \leqslant N} B_{n} \frac{(\ln x)^{\frac{2 k}{D}-n-1}}{\Gamma\left(\frac{2 k}{D}-n\right)}+O\left(x(\ln x)^{\frac{2 k}{D}-N-2}\right) .
$$

ДОКАЗАТЕЛЬСТВО следствий 1 и 2 состоит в непосредственном применении теоремы 1 для случая $\alpha_{l_{1}}=\cdots=\alpha_{l_{k}}=1$ или для случая $\alpha_{l_{1}}=\cdots=\alpha_{l_{k}}=2$, причем все прочие $\alpha_{i}$ равны нулю. Коэффициенты $A_{n}$ и $B_{n}$ суть коэффициенты Тейлора соответствуюших функций $H(s)$.

Заметим, что константы $A_{0}$ и $B_{0}$ в следствиях 1 и 2 можно представить в виде бесконечных произведений по простым числам. Действительно, потенцируя соотношение (2) при $s=1$ и переходя к пределу при $x \rightarrow \infty$, в силу оценки (1) получаем

$$
e^{G_{l}(1)}=\prod_{p}\left(1-\frac{1}{p}\right)^{\frac{1}{D}-\delta_{l}(p)} .
$$

Отсюда, ограничиваясь первым членом, находим

$$
\sum_{\substack{n \leq x \\ n \in M}} 1=C x \frac{(\ln x)^{\frac{k}{D}-1}}{\Gamma\left(\frac{k}{D}\right)}+O\left(x(\ln x)^{\frac{k}{D}-2}\right)
$$

и

$$
\sum_{\substack{n \leqslant x \\ n \in M}} \tau(n)=C^{2} x \frac{(\ln x)^{\frac{2 k}{D}-1}}{\Gamma\left(\frac{2 k}{D}\right)}+O\left(x(\ln x)^{\frac{2 k}{D}-2}\right)
$$

где

$$
C=\prod_{p}\left(1-\frac{1}{p}\right)^{\frac{k}{D}-\delta_{l_{1}}(p)-\cdots-\delta_{l_{k}}(p)}=e^{G_{l_{1}}(1)+\cdots+G_{l_{k}}(1)}>0 .
$$

Если в асимптотической формуле теоремы $1 \alpha$ кратно $D$, то сумма с растущим числом слагаемых в главном члене обращается в конечную сумму. Например, при $D=2$ имеем

$$
\sum_{\substack{n \leqslant x \\ n \in M_{1}}} \tau(n)=C^{2} x+O\left(x e^{-c \sqrt{\ln x}}\right),
$$

где $C>0$ выражается формулой (9) при $k=1$ и $l_{1}=1$. Аналитическая природа этого эффекта, уже обсуждавшаяся в замечании 1 , заключается в однозначном 
характере особой точки соответствующей производящей функции. Тот же эффект возникает и при рассмотрении чисел, все простые делители которых лежат в заданных арифметических прогрессиях. Такая задача была впервые рассмотрена Э. Ландау [8], а в более общей постановке - в работах [9], [10].

Остановимся еше на одном проявлении того же эффекта. Известно, что

$$
\sum_{n \leqslant x} \mu(n)=O\left(x e^{-c \sqrt{\ln x}}\right) .
$$

Из следуюшей теоремы становится понятно, что если вести суммирование только по $n$, принадлежашим множеству $M$, то возникает главный член, имеющий вид суммы с растушим числом слагаемых.

TEOPEMA 2. Пусть

$$
H(s)=\frac{1}{s}((s-1) \zeta(s))^{-\frac{k}{D}} e^{-G_{l_{1}}(s)-\cdots-G_{l_{k}}(s)},
$$

причем функция $H(s)$ положительна при $s>1, A_{n}$ обозначает $n$-й коэффициент ее разложения Тейлора с центром в точке $s=1$. Тогда имеет место асимптотическая формула

$$
\sum_{\substack{n \leqslant x \\ n \in M}} \mu(n)=x \sum_{0 \leqslant n \leqslant \sqrt{\ln x}} A_{n} \frac{(\ln x)^{-\frac{k}{D}-n-1}}{\Gamma\left(-\frac{k}{D}-n\right)}+O\left(x e^{-c \sqrt{\ln x}}\right) .
$$

Кроме того, при любом фиксированном иелом $N$

$$
\sum_{\substack{n \leqslant x \\ n \in M}} \mu(n)=x \sum_{0 \leqslant n \leqslant N} A_{n} \frac{(\ln x)^{-\frac{k}{D}-n-1}}{\Gamma\left(-\frac{k}{D}-n\right)}+O\left(x(\ln x)^{-\frac{k}{D}-N-2}\right) .
$$

ДокАЗАТЕЛЬСТво. В области $\operatorname{Re} s>1$ производящая функция задается равенством

$$
F(s)=\sum_{\substack{n=1 \\ n \in M}}^{\infty} \frac{\mu(n)}{n^{s}}=F_{l_{1}}^{-1}(s) \ldots F_{l_{k}}^{-1}(s) .
$$

Возьмем параметр $b=1+1 / \ln x$. Используя общую теорему о связи между суммой коэффициентов ряда Дирихле и функцией, задаваемой этим рядом $[7$, с. 75$]$, имеем

$$
\sum_{\substack{n \leqslant x \\ n \in M}} \mu(n)=\frac{1}{2 \pi i} \int_{b-i T}^{b+i T} F(s) \frac{x^{s}}{s} d s+O\left(x e^{-c_{11} \sqrt{\ln x}}\right),
$$

где $T=e^{c_{1} \sqrt{\ln x}}, c_{1}-$ постоянная из леммы 1 . Дальнейшие преобразования комплексного интеграла в правой части полностью аналогичны таковым при доказательстве теоремы 1.

Константу $A_{0}$ в теореме 2 также удобно представить в виде бесконечного произведения. Ограничиваясь первым членом, находим

$$
\sum_{\substack{n \leqslant x \\ n \in M}} \mu(n)=C^{-1} x \frac{(\ln x)^{-\frac{k}{D}-1}}{\Gamma\left(-\frac{k}{D}\right)}+O\left(x(\ln x)^{-\frac{k}{D}-2}\right),
$$

где $C>0$ выражается формулой (9). 


\section{Список литературы}

1. Виноградов И. М. Некоторое общее свойство распределения простых чисел // Матем. сб. 1940. Т. 7 (49). №2. С. 365-372.

2. Leitmann D. On the uniform distribution of some sequences // J. London Math. Soc. 1976. V. 14. P. 430-432.

3. Baker R. C., Kolesnik G. On the distribution of $p^{\alpha}$ modulo one // J. Reine Angew. Math. 1985. V. 356. P. 174-193.

4. Чанга M.E. Простые числа в специальных промежутках и аддитивные задачи с такими числами // Матем. заметки. 2003. Т. 73. № 3. С. 423-436.

5. Титчмари E. К. Теория дзета-функции Римана. М.: ИЛ, 1953.

6. Уиттекер Э.Т., Ватсон Дж. Н. Курс современного анализа. Т. 2. М.: Физматгиз, 1963.

7. Карацуба А. А. Основы аналитической теории чисел. М.: Наука, 1983.

8. Landau E. Handbuch der Lehre von der Verteilung der Primzahlen. V. 2. Leipzig: Teubner, 1909.

9. Бредихин Б. М. Остаточный член в асимптотической формуле для $\nu_{G}(x) / /$ Изв. вузов CССР. Математика. 1960. Т. 6(19). С. 40-49.

10. Левин Б. В., Файнлейб А. С. Применение некоторых интегральных уравнений к вопросам теории чисел // УМН. 1967. Т. 22. №3 (135). С. 119-198.

Поступило в редакцию

18.II.2003 